\title{
Neutrinoless Double-Beta becay With EXO: Achievements And Prospects
}

\section{Tamer Tolba*t}

Institute of High Energy Physics, Chinese Academy of Sciences

E-mail: tolba@ihep.ac.cn

In this report, we discuss the latest EXO-collaboration results in the search for the $2 v \beta \beta$ and the $0 v \beta \beta$ decays of the Xenon, enriched to $80.67 \%{ }^{136} \mathrm{Xe}$ isotope. We will also discuss the R\&D design effort and philosophy for the future ton-scale experiment, known as nEXO, in decrease background, increase energy resolution and overall detector sensitivity.

The 26th International Nuclear Physics Conference

11-16 September, 2016

Adelaide, Australia

\footnotetext{
* Speaker.

On behalf of the EXO-200 and nEXO Collaborations.
} 


\section{Introduction}

In the search for the nature of the neutrino, neutrinoless double beta decay $(0 v \beta \beta)$ plays a significant role in understanding its properties. By measuring the $(0 v \beta \beta)$ decay rate, with the desired sensitivity, it is hoped to verify the nature of the neutrino (Majorana or Dirac particle), lepton number violation and help determine the values for the absolute neutrino masses. The $0 v \beta \beta$ decay is the hypothetical decay mode of the rare double beta decay process (that occurs if the single beta decay is either energetically forbidden or highly spin suppressed [1]), while the $2 v \beta \beta$ decay is the other decay mode. The $2 v \beta \beta$ mode is allowed in the standard model (SM) as a second-order weak process, where the two emitted electrons, in the final state, are accompanied by the emission of two anti-neutrinos. While the $2 v \beta \beta$ decay mode has been observed in many isotopes with halflives ranging between $10^{18}$ and $10^{21}$ years [2], the $0 v \beta \beta$ mode has not been observed yet.

The $0 v \beta \beta$ decay rate is related to the square of the effective Majorana neutrino mass $\left\langle m_{\beta \beta}\right\rangle$ by the product of a phase space factor and a nuclear matrix element square. Kinematic measurements restrict the neutrino mass scale to be below $O(1 \mathrm{eV})$ [3], leading to $0 v \beta \beta$ half-lives beyond $10^{24}$ years. The two decay modes are distinguishable by studying the sum energy spectrum of the two released electrons in the final state. While in case of $2 v \beta \beta$ decay the two electrons have sum energies covering the range from threshold to the Q-value $(2457.83 \pm 0.37) \mathrm{keV}$, the $0 v \beta \beta$ decay shows a delta peak at the Q-value of the decay. Hence, the $0 v \beta \beta$ signal can be easily hidden in the high energy tail of the $2 v \beta \beta$ broad energy distribution. Therefore, the future multi-tonne nEXO R\&D program aims for the development of an ultra-low background and high energy resolution experiment that aims at raising the $0 v \beta \beta$ half-life sensitivity to $10^{27}$ years (at $90 \%$ C.L.). This value is sufficient to achieve $\left\langle m_{\beta \beta}\right\rangle$ at the bottom of the inverted hierarchy, for a wider range of nuclear matrix elements. Recent searches carried out with ${ }^{76} \mathrm{Ge}$ (the GERDA experiment) [4] and ${ }^{136}$ Xe (the KamLAND-Zen [5] and EXO-200 experiments [6]) have established the lifetime of this decay to be longer than $10^{25}$ years, corresponding to a limit on the neutrino mass of 0.06-0.4 electronvolts.

\section{EXO-200}

The EXO-200 detector, discussed in detail in [7], uses xenon both as source and detector for the two electrons emitted in its $\beta \beta$ decay. The detector is a cylindrical homogeneous time projection chamber (TPC). It is filled with liquefied xenon (enrLXe) enriched to $(80.672 \pm 0.14) \%$ in the isotope ${ }^{136} \mathrm{Xe}$. The remaining $19.328 \%$ is ${ }^{134} \mathrm{Xe}$, with other isotopes present only at low concentration. EXO-200 is designed to minimize radioactive backgrounds, maximize the (enrLXe) fiducial volume, and provide good energy resolution at the ${ }^{136} \mathrm{Xe}$ Q-value [8]. Energy depositions in the TPC produce both ionization and scintillation signals. The TPC configuration allows for three-dimensional topological and temporal reconstruction of individual energy depositions. This ability is essential for discriminating $\beta \beta$ decays from residual backgrounds dominated by $\gamma_{\mathrm{s}}$.

The cylindrical TPC is divided into two symmetric volumes separated by a cathode grid. Each end of the TPC is instrumented with 38 charge induction (V) and 38 charge collection (U) wire triplets. The $\mathrm{U}$ and $\mathrm{V}$ wire grids, crossing at $60 \mathrm{deg}$, provide stereoscopic information for charge depositions. At each end of the TPC there are 250 Large Area Avalanche Photodiodes (LAAPDs) [9] 
that record the $178 \mathrm{~nm}$ scintillation light. A drift field of $376 \mathrm{~V} / \mathrm{cm}$ is applied in the TPC volume. All signals are digitized at MS/s. All detector components were carefully selected to minimize internal radioactivity. The TPC is mounted in the center of low-background cryostat. The TPC vessel is surrounded by a $50 \mathrm{~cm}$ thick thermal bath of HFE-7000 cryofluid [10], which maintains the temperature of the TPC and shields the detector from external gamma radiation. The HFE-7000 is housed in a double-walled vacuum-insulated cryostat composed of two nested copper vessels, fabricated from low-radioactivity copper plate of $27 \mathrm{~mm}$ thickness. The outer cryostat is surrounded in all directions by at least by $25 \mathrm{~cm}$ of lead. The cryostat features a copper guide tube which allows radioactive sources to be inserted past the lead shield and into the cold HFE volume near the detector. ${ }^{137} \mathrm{Cs},{ }^{60} \mathrm{Co}$ and ${ }^{228} \mathrm{Th}$ sources of various intensities are available for deployment.

The entire assembly is located in a class 100 clean-room which is surrounded on four of six sides by a cosmic ray veto system. The veto system consists of twenty-nine $5 \mathrm{~cm}$ thick Bicron BC412 plastic scintillator panels obtained from the concluded KARMEN neutrino experiment [11]. Each panel is observed by eight photomultiplier tubes (PMTs) and is supported by $4 \mathrm{~cm}$ of borated polyethylene. The clean-room is installed underground at the Waste Isolation Pilot Plant (WIPP) near Carlsbad, New Mexico, USA providing 1585 meters water equivalent of over burden [12].

\subsection{EXO-200 Phase-I}

\subsection{1 $2 v \beta \beta$ mode}

EXO-200 started Phase-I data collection in May 2011 until February 2014, when unexpected underground accidents at WIPP forced all operations to be suspended temporarily [13]. With the analysis of the data collected between September 2011 and April 2012 (run 2a) with total exposure of $23.14 \mathrm{~kg} \cdot$ year, EXO-200 published a precision measurement of ${ }^{136} \mathrm{Xe}$ half-life, $T_{1 / 2}^{2 v \beta \beta}=$ $(2.165 \pm 0.016$ (stat) \pm 0.059 (sys) $) \times 10^{21}$ years [14], the most precise $T_{1 / 2}^{2 v \beta \beta}$ of any isotope.

\subsection{2 $0 v \beta \beta$ mode}

Using the low-background data collected in the first two years of its Phase-I operation and total ${ }^{136} \mathrm{Xe}$ exposure of $100 \mathrm{~kg} \cdot$ year, EXO-200 published a $0 v \beta \beta$ decay half-life limit $T_{1 / 2}^{0 v \beta \beta}>1.1 \times 10^{25}$ years (consistent with the null hypothesis at $90 \%$ C.L.) [6]. This result corresponds to a limit on the Majorana neutrino mass $\left\langle m_{\beta \beta}\right\rangle$ of $190-450 \mathrm{meV}$ (for a range of nuclear matrix elements). The background rate at the region of interest ROI $(Q$-value $\pm 2 \sigma)$ found to be $(1.7 \pm 0.2) \times 10^{-3}$ $\mathrm{keV}^{-1} \mathrm{~kg}^{-1} \mathrm{yr}^{-1}$.

\subsection{EXO-200 Phase-II}

EXO-200 resumed underground operation in early 2015. After initial inspection, serious equipment damage has been found, including power supplies and refrigerators. Two major detector upgrades were carried out between February and April 2016. First, the frontend readout system was upgraded to reduce coherent noise in the scintillation channels and lower the threshold for the $\mathrm{V}$ wire channels. Second, a Rn-suppressed air system was commissioned to purge the air gap between the cryostat and lead shielding. EXO-200 started Phase-II low background data collection on Apr. 2016 (with expectations to continue data taking until 2018). The sensitivity studies 
indicate that with the detector upgrades and analysis improvements, EXO-200 Phase-II can reach a $0 v \beta \beta$ decay half-life sensitivity of $5.7 \times 10^{25}$ years.

\section{3. nEXO}

It has been proven that currently running $\mathrm{kg}$-scale experiments are able to explore the inverted neutrino mass hierarchy $[5,6]$. On the other hand, and in order to be able to resolve "with high confidence" the issue of Majorana vs. Dirac nature of the neutrinos for the inverted hierarchy, the next-generation experiments must have a half-life sensitivity significantly higher than $10^{27}$ years. nEXO is the improved tonne-scale version of the EXO-200 detector, dedicated to cover the entire range of the inverted mass hierarchy, with unique possibilities to explore the normal mass hierarchy (with $T_{1 / 2}^{0 v \beta \beta}>10^{28}$ years) when equipped with the Ba-tagging technique[15].

\section{1 nEXO R\&D}

With the experience gained from constructing and running the EXO-200 detector, the nEXO collaboration started a comprehensive $R \& D$ program to validate new detector concepts. nEXO will be a modified version of EXO-200, therefore its R\&D program depends on optimizations of the EXO-200 technologies, beside the new demands that are required by the new design and the physics case of the nEXO experiment.

\subsubsection{Charge readout technique}

The ionization electrons will be collected by a segmented anode made of $10 \times 10 \mathrm{~cm}^{2}$ "tiles" (Figure 1 (left)). Each tile includes an array of crossed strips with a shape optimized to cover the substrate as much as possible. The use of a fully 2-dimensional pad structure was not required, because of the low event multiplicity (and the extra complexity due to the very large channel count). In this way, the anode has no Frisch grid. This design allows to:

- Eliminate the need for a bulky wire tensioning frame and

- Reduce the $x-y$ position reconstruction ambiguity affecting large-area, crossed-wire designs.

In contrast to crossed wires (as in the case of EXO-200), however, this anode design is opaque and does not allow for photosensors to be installed behind it, a fact that together with the absence of a side PTFE reflector makes it necessary to install photodetectors on the TPC lateral surface behind the field cage. This configuration also provides a larger photon collection area (as will be explained in the next section).

This new readout technique has been developed by the EXO-IHEP/IME group in China. The first prototypes, with strips size of $3 \mathrm{~mm}$ and interstrip spacing of $\approx 500 \mu \mathrm{m}$, have been tested in a LXe (liquid Xenon) cell with ${ }^{207} \mathrm{Bi} \gamma$-source, by the EXO-Stanford group in the USA. Figure 1 (right), shows the deposited energy spectrum (blue shaded area) compared to the Monte-Carlo simulations (red shaded area). The spectrum shows a charge readout tile to the ${ }^{207} \mathrm{Bi}$ source placed on the cathode. A drift-time cut has been applied to select the region of the detector where the Electric field is uniform, and a single-channel cut was used to select events that only deposited energy in one channel. Currently, the best energy resolution achieved for the $570 \mathrm{keV}$ peak $\frac{\sigma}{E}=5.7 \%$, compared 

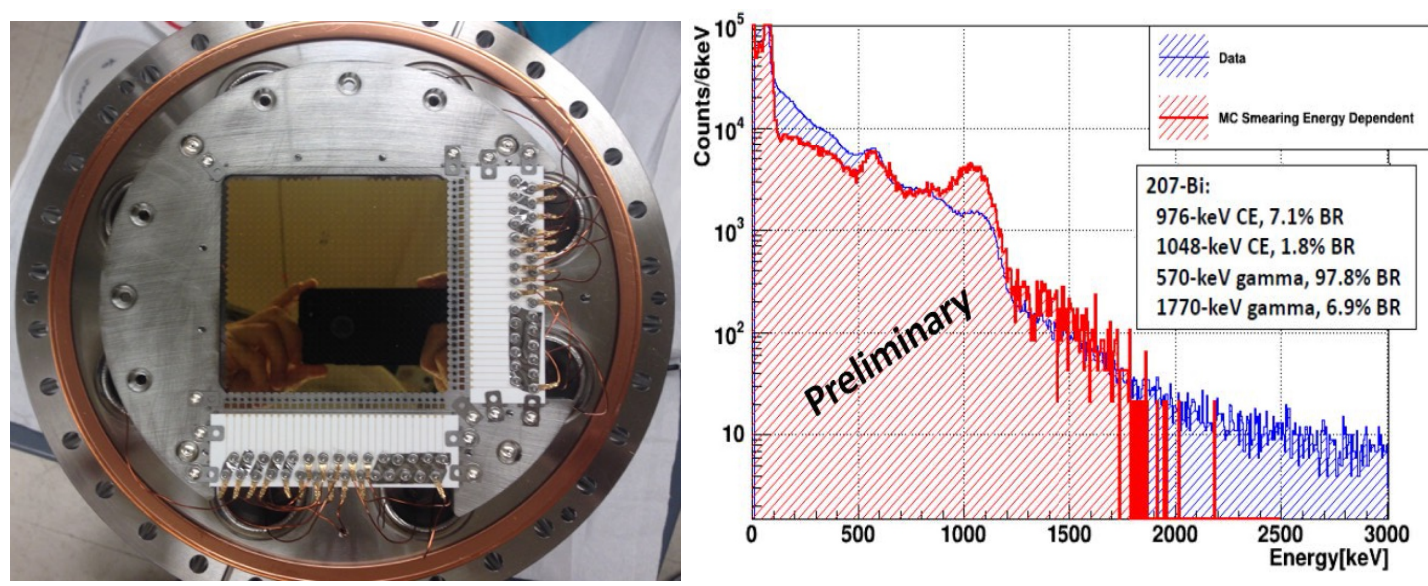

Figure 1: (Left) $10 \times 10 \mathrm{~cm}^{2}$ tile, tested at Stanford University. (Right) The deposited energy spectrum (blue shaded area) compared to the Monte-Carlo simulations (red shaded area) for the $10 \times 10 \mathrm{~cm}^{2}$ tile.

to $5.4 \%$ predicted by the Monte Carlo simulation. This resolution extrapolates to better than $1 \%$ at the Q-value, when the the scintillation light is included with the appropriate nEXO parameters.

\subsubsection{Light readout technique}

Efficient detection of LXe scintillation photons is critical to achieve the desired energy resolution of $\frac{\sigma_{E}}{E}=1 \%$ or better. The current $\mathrm{nEXO}$ concept has an array of silicon photomultipliers (SiPMs) located behind the field shaping rings, a configuration that provides better coverage (4-5 $\mathrm{m}^{2}$ ) than the EXO-200 option, where the Avalanche PhotoDiodes (APDs) were located behind the anode planes.

Although, during the past decad, substantial development in the area of SiPMs has offered what appears to be a superior alternativeto conventional methods for our detector, SiPMs are still counted as relatively new technology. Hence, not all their features have been examined under the influence of extreme working environments. Although, it is known that the SiPMs are stable against the change in the magnetic field, but little is known about their behavior in high electric field variations.

In the current design of the nEXO field cage, the SiPMs will be exposed to different electric field values along the drift axis. The preliminary electrostatic simulations show that the SiPMs will be exposed to electric field values as high as $30 \mathrm{kV} / \mathrm{cm}$ at the regions close to the cathode plane and the few rings above it, and to much lower values as it goes toward the anode. Moreover, the SiPM chips can act field discontinuities on the grounded SiPMs support plate. This can produce regions with very high local surface field values, leading to discharges. There are serious concerns that such exposure might lead to:

1. Physical damage to the body of the sensor and its readout electronics, due to the electric overstresses caused by the high E fields.

2. Change in the SiPM properties (gain, dark noise, cross-talks, etc.).

3. Charge build up/charge injection on the $\mathrm{SiO}_{2}$ layer on the surface of the SiPM chips. 
Figure 2 (left) shows a collection of SiPMs considered for nEXO. Figure 2 (right) shows the results of a collection of electro-optic measurements of these SiPMs. In this context the photodetection efficiency (PDE) is defined as the probability of detecting photons hitting the device (i.e. the product of the quantum efficiency and the geometrical fill factor on one die).
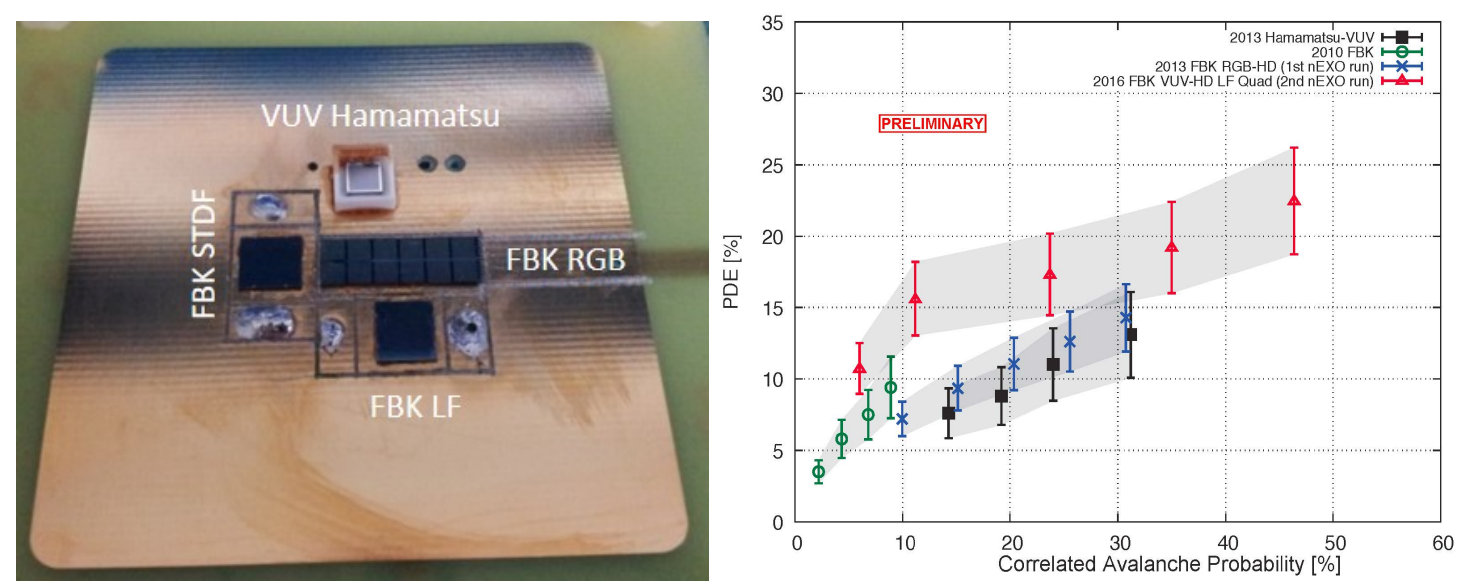

Figure 2: (Left) A collection of SiPMs considered for nEXO. (Right) the results of a collection of electrooptic measurements of the SiPMs.

Figure 3 shows preliminary results for the ADC counts as a function of electric field values (for a range between $0 \mathrm{kV} / \mathrm{cm}$ to $30 \mathrm{kV} / \mathrm{cm}$ ). Two sets of data have been collected; a) the light signal using a blue light LED-source $(460 \mathrm{~nm}), \mathrm{b})$ the dark current signal. The results shown here are only for the studies with UV light source. This test has been reapeated two times, in liquefied $\mathrm{CF}_{4}(-125$ ${ }^{\circ} \mathrm{C}$ ), for double checking the results. The totoal charge of the wave-forms has been calculated and normlized (event by event) to the charge collected by a monitor PMT and then fitted with guassian fit. The mean of the fit was used as the mean value of the total charge, which is then normalized to the total charge collected with $0 \mathrm{kV} / \mathrm{cm}$ electric field measurement.

The results obtained from the HV tests, shows stability in the different types of SiPMs performance when they are exposed to high electric field values. Also physical inspections were performed on the body of the SiPM chips. The SiPM chips used in these tests are bare chips except for the Hamamatsu one, which was in a special carrier made by the company. No evidences of strong discharges on the body of the chips were found, indicating that the SiPMs chips are safe to be used in the high electric fields environment.

\section{Conclusion}

EXO-200 Phase-I has reported on the most precise $T_{1 / 2}^{2 v \beta \beta}$ of any isotope. EXO-200 also reported measurements of $0 v \beta \beta$ decay in ${ }^{136} \mathrm{Xe}$ with effective Majorana masses of less than 190 $450 \mathrm{meV}$. EXO-200 operation Phase-II is taking data now with the aim of reaching a $0 v \beta \beta$ decay half-life sensitivity of $5.7 \times 10^{25}$ years by the end of this phase. We also reported on the various $\mathrm{R} \& \mathrm{D}$ efforts in the preparation for the next generation 5-tonne detector nEXO, with particular focus 

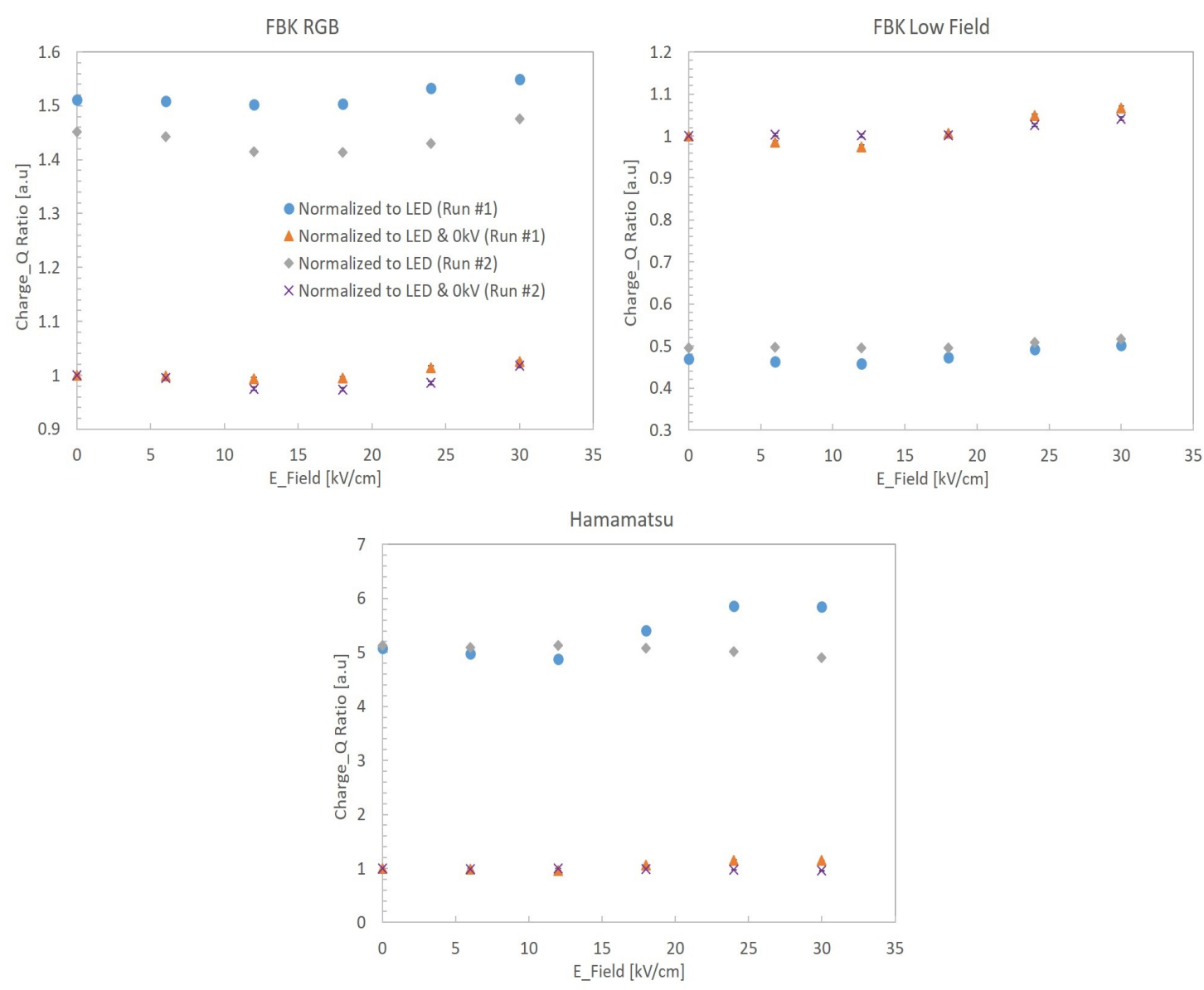

Figure 3: Total charge collected at the SiPMs normalized to the charge collected by the monitor PMT and by the $0 \mathrm{kV} / \mathrm{cm}$ electric field measurement.

on the new methodes for charge and light collection techniques, which are intended to be used in nEXO to improve the energy resoultion of the detector.

\section{References}

[1] I. Ogawa et al., J. Phys.: Conf. Ser. 375, (2012) 042018.

[2] J. beringer et al., (Particle Data Group), Phys. Rev. D 86, (2012) 010001.

[3] V.N. Aseev et al., Phys. Rev. D 84, (2011) 112003.

[4] M. Agostini et al., Nucl. Part. Physics Procs. 273 - 275 (2016) 1876.

[5] A. Gando et al., Phys. Rev. Lett. 117 (2016) 109903.

[6] J. B. Albert et al., Nature 510, (2014) 229.

[7] M. Auger et al., JINST 7, (2012) P05010.

[8] M. Redshaw et al., Phys. Rev. Lett. 98, (2007) 053003.

[9] R. Neilson et al., Nucl. Instrum. Methods Phys. Res., Sect. A 608, (2009) 68. 
[10] 3M, see http://www.3m.com/product/index.html.

[11] H. Gemmeke et al., Ncul. Inst. \& Meth. A 289, (1990) 490.

[12] E.-I. Esch et al., Nucl. Inst. \& Meth. A 538, (2005) 516.

[13] WIPP, see http://wipp.energy.gov/wipprecovery/accident_desc.html

[14] J. B. Albert et al., Phys. Rev. C89 (2014) 015502.

[15] K. Twelker et al., Rev. Sci. Instrum. 85, (2014) 095114; 10.1063/1.4895646 